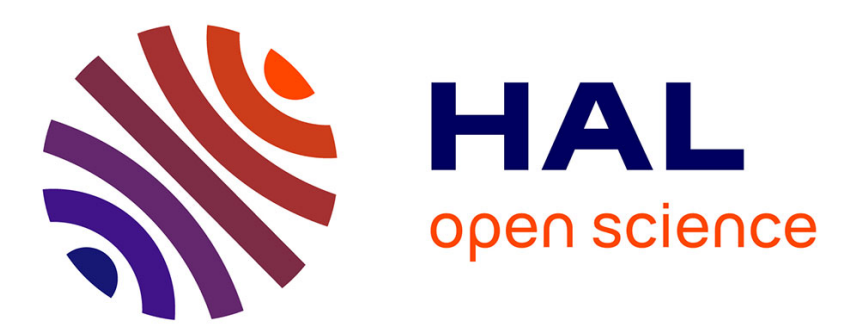

\title{
Terahertz Kerr effect in gallium phosphide crystal
}

\author{
Marion Cornet, Jérôme Degert, Emmanuel Abraham, Eric Freysz
}

\section{To cite this version:}

Marion Cornet, Jérôme Degert, Emmanuel Abraham, Eric Freysz. Terahertz Kerr effect in gallium phosphide crystal. Journal of the Optical Society of America B, 2014, 31 (7), pp.1648-1652. 10.1364/JOSAB.31.001648 . hal-01058587

\section{HAL Id: hal-01058587 https://hal.science/hal-01058587}

Submitted on 27 Aug 2014

HAL is a multi-disciplinary open access archive for the deposit and dissemination of scientific research documents, whether they are published or not. The documents may come from teaching and research institutions in France or abroad, or from public or private research centers.
L'archive ouverte pluridisciplinaire HAL, est destinée au dépôt et à la diffusion de documents scientifiques de niveau recherche, publiés ou non, émanant des établissements d'enseignement et de recherche français ou étrangers, des laboratoires publics ou privés. 


\title{
Terahertz Kerr effect in gallium phosphide crystal
}

\author{
M. Cornet, ${ }^{1,2}$ J. Degert ${ }^{1,2, *}$ E. Abraham, ${ }^{1,2}$ and E. Freysz ${ }^{1,2}$ \\ ${ }^{1}$ Université de Bordeaux, Laboratoire ondes et matière d'Aquitaine (LOMA), Unité mixte de recherche (UMR) 5798, \\ F-33400 Talence, France \\ ${ }^{2}$ Centre national de la recherche scientifique (CNRS), Laboratoire ondes et matière d'Aquitaine (LOMA), \\ Unité mixte de recherche (UMR) 5798, F-33400 Talence, France \\ *Corresponding author: j.degert@loma.u-bordeaux1.fr
}

Received April 4, 2014; revised May 19, 2014; accepted May 22, 2014;

posted May 22, 2014 (Doc. ID 209591); published June 23, 2014

We report on the observation of the Kerr effect induced by an intense terahertz pulse in a $\langle 100\rangle$ gallium phosphide crystal. The temporal and angular behaviors of the phase retardation have been measured and agree well with theoretical predictions. From these measurements, we extracted the two nonzero tensor elements of the thirdorder response function of the crystal in the terahertz range. @ 2014 Optical Society of America OCIS codes: $\quad$ (190.3270) Kerr effect; (300.6495) Spectroscopy, terahertz. http://dx.doi.org/10.1364/JOSAB.31.001648

\section{INTRODUCTION}

Discovered in 1875, the Kerr effect corresponds to a modification of the refractive index of a medium induced by an external electric field. With the advent of the laser in the 1960 s, the lightinduced Kerr effect has enabled important breakthroughs in the field of ultrafast optics [1]. Among them, one can cite the realization of stable and reliable laser sources emitting ultrashort light pulses of just a few femtoseconds in duration and the revolution of telecommunication through fiber-optic communication. Moreover, the Kerr effect induced by ultrashort light pulses is also a good tool for probing the dynamical response of transparent media on femtosecond time scales [2]. For a while, these experiments were limited to the study of the optical properties of materials in the visible and infrared ranges due to lack of appropriate sources in other spectral ranges. However, thanks to recent developments of intense pulsed sources in the terahertz (THz) range, the THz-induced Kerr effect (TKE) has been reported in isotropic media such as liquids [3] and glasses [4], whereas self-phase modulation of single-cycle $\mathrm{THz}$ pulses was reported in lithium niobate [5] and bulk $n$-GaAs [6]. Hereafter we report on the observation of TKE in a gallium phosphide (GaP) crystal, the latter being widely used in the $\mathrm{THz}$ range owing to its second-order nonlinear optical properties. Such a crystal is expected to have a nonlinear refraction coefficient one order of magnitude greater than the ones of liquids or glasses. Moreover, since electro-optic detection of intense $\mathrm{THz}$ pulses in zinc blende crystals may be distorted by the Kerr-like quadratic electrooptic effect, the study of the THz Kerr effect in GaP deserves some attention. Finally, at variance with previous TKE studies performed in amorphous media [3,4] , our experiment takes place in a cubic crystal (point group $\overline{4} 3 \mathrm{~m}$ ). As a consequence, the nonlinear crystal response is expected to depend upon its orientation with respect to the $\mathrm{THz}$ wave polarization, and this must be taken into account in the analysis of the experiment.

\section{EXPERIMENTAL SETUP}

The experiment is as follows [Fig. 1(a)]: an intense linearly polarized $\mathrm{THz}$ pulse spanning the $0 . \overline{3-7} \mathrm{THz}$ frequency range is emitted from air ionized by a two-color (namely, 400 and $800 \mathrm{~nm}$ ) femtosecond laser field [7]. This THz pump field is then collimated and focused onto the sample by two off-axis paraboloidal mirrors with a $150 \mathrm{~mm}$ focal length. There its amplitude, $E_{\mathrm{THz}}$, reaches $80 \mathrm{kV} / \mathrm{cm}$. The sample consists in either a $300 \mu \mathrm{m}$ or a $1 \mathrm{~mm}$ thick $\langle 100\rangle \mathrm{GaP}$ crystal. For such cut, it does not exhibit any Pockels effect or second-order response. Inside these crystals, the $\mathrm{THz}$ beam and a weak $800 \mathrm{~nm}$ probe beam, initially polarized linearly at $45^{\circ}$ with respect to the $\mathrm{THz}$ polarization, propagates collinearly along the $\langle 100\rangle$ axis [Fig. 1(b)]. Note that, to set the polarization of the probe pulse, we used a Babinet-Soleil compensator instead of a half-wave plate in order to correct some depolarizing effects due to the pellicle. The birefringence induced by the THz pump alters the polarization state of the probe pulse, the latter being analyzed by means of an ellipsometer consisting in a quarter-wave plate combined with a Wollaston prism, followed by two balanced photodiodes connected to a lock-in amplifier for detection. The crystal is inserted into a rotation mount, enabling us to rotate it by an angle $\theta$ (the so-called azimuthal angle) perpendicularly to the $\langle 100\rangle$ axis [Fig. 1(b)]. A delay stage makes it possible to delay the probe pulse with respect to the THz pump pulse. Finally, a pair of high-density polyethylene polarizers inserted on the path of the $\mathrm{THz}$ beam enables us to attenuate the latter.

\section{THEORETICAL MODEL}

To model this experiment, let us write

$$
E_{\mu}(t, x)=\frac{1}{2} \mathcal{E}_{\mu}(t, x) \exp \left[i\left(k_{\mathrm{pr}} x-\omega_{\mathrm{pr}} t\right)\right]+\text { c.c. },
$$

the field of the probe pulse in the frame of the laboratory, and

$$
P_{\mu}^{(3)}(t, x)=\frac{1}{2} \mathcal{P}_{\mu}^{(3)}(t, x) \exp \left[i\left(k_{\mathrm{pr}} x-\omega_{\mathrm{pr}} t\right)\right]+\text { c.c. }
$$




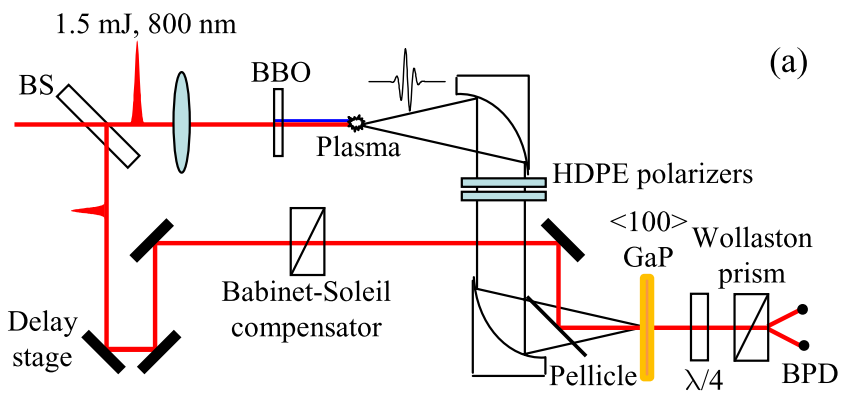

(b)

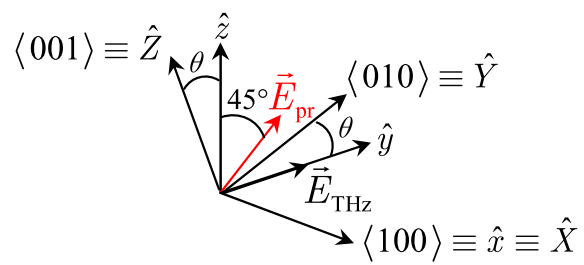

Fig. 1. (a) Experimental setup. BS, beam splitter; BPD, balanced photodiodes. (b) Geometry of the experiment. $(\hat{X}, \hat{Y}, \hat{Z})$, Cartesian frame of the crystal; $(\hat{x}, \hat{y}, \hat{z})$, Cartesian frame of the laboratory; $\theta$, azimuthal angle.

the third-order polarization of the crystal $(\mu=y, z)$. Here $\omega_{\mathrm{pr}}$ and $k_{\mathrm{pr}}$ stand for the angular frequency and the wave vector of the probe beam. Then, within the slowly varying amplitude approximation, the probe wave equation writes [8]

$$
\frac{\partial \mathcal{E}_{\mu}\left(t^{\prime}, x\right)}{\partial x}=i \frac{\omega_{\mathrm{pr}}^{2}}{2 k_{\mathrm{pr}} \varepsilon_{0} c^{2}} \mathcal{P}_{\mu}^{(3)}\left(t^{\prime}, x\right)
$$

where $t^{\prime}=t-x / v_{g}, c$ is the velocity of light in vacuum, $\varepsilon_{0}$ is the permittivity of vacuum, and $v_{g}$ is the group velocity of the probe pulse.

In the frame of the crystal, assuming that we are far from resonance and writing the real-valued $\mathrm{THz}$ electric field as $E_{\mathrm{THz}}(t, x)=(1 / 2) E_{\mathrm{pu}}(t, x)$, the third-order polarization is given by [8]

$$
\begin{aligned}
\mathcal{P}_{i}^{(3)}\left(t^{\prime}, x\right)= & \frac{3 \varepsilon_{0}}{4} \sum_{j, k, l} \mathcal{R}_{i j k l}^{(3)} \mathcal{E}_{j}\left(t^{\prime}, x\right) \\
& \times E_{\mathrm{pu}, k}\left(t^{\prime}+\frac{x}{v_{g}}, x\right) E_{\mathrm{pu}, l}\left(t^{\prime}+\frac{x}{v_{g}}, x\right),
\end{aligned}
$$

where $\mathcal{R}_{i j k l}^{(3)}$ is the third-order response function $(i, j, k, l=X, Y, Z)$. As emphasized by Gallot and Grischkowsky concerning the electro-optic detection in [9], the phase retardation induced on the optical pulse by the $\mathrm{THz}$ wave can be understood as "the generation of phase-coherent sidebands on the spectrum of the optical pulse through the generation of sum- and difference-frequency components" by interaction with the $\mathrm{THz}$ wave. This line of thought applies as well to the Kerr effect considered here. That is why we are using the real THz electric field in Eq. (2) instead of its complex representation to account fully for the generation of these sidebands. Owing to the symmetry of the crystal, there are only two independent, nonzero tensor elements of the third-order response function: $a=\mathcal{R}_{\text {iiii }}^{(3)}$ and $b=\mathcal{R}_{i i j j}^{(3)}=\mathcal{R}_{i j j i}^{(3)}=\mathcal{R}_{i j i j}^{(3)}$.
In the frame of the laboratory, $\mathcal{P}^{(3)}$ becomes

$$
\mathcal{P}_{\mu}^{(3)}\left(t^{\prime}, x\right)=\frac{3 \varepsilon_{0}}{4} \delta \mathcal{R}_{\mu \mu}\left(t^{\prime}+\frac{x}{v_{g}}, x\right) \mathcal{E}_{\mu}\left(t^{\prime}, x\right)
$$

with

$$
\begin{aligned}
& \delta \mathcal{R}_{y y}(t, x)=b\left[u-\frac{u-3}{2} \sin ^{2} 2 \theta+\frac{u-3}{4} \sin 4 \theta\right] I_{\mathrm{pu}}(t, x), \\
& \delta \mathcal{R}_{z z}(t, x)=b\left[1+\frac{u-3}{2} \sin ^{2} 2 \theta+\frac{u-3}{4} \sin 4 \theta\right] I_{\mathrm{pu}}(t, x),
\end{aligned}
$$

where $u=a / b$ and $I_{\mathrm{pu}}(t, x)=E_{\mathrm{pu}}^{2}(t, x)$.

Thanks to Eq. (3), Eq. (1) leads to

$$
\mathcal{E}_{\mu}\left(t^{\prime}, L\right)=\mathcal{E}_{\mu}\left(t^{\prime}, 0\right) \exp \left[i \phi_{\mu}\left(t^{\prime}, L\right)\right],
$$

with

$$
\phi_{\mu}\left(t^{\prime}, L\right)=\frac{3 \pi}{4 \lambda_{\mathrm{pr}} n_{\mathrm{pr}}} \int_{0}^{L} \delta \mathcal{R}_{\mu \mu}\left(t^{\prime}+\frac{x}{v_{g}}, x\right) \mathrm{d} x,
$$

$\lambda_{\text {pr }}$ and $n_{\text {pr }}$ being, respectively, the wavelength and the refractive index of the probe pulse, and $L$ is the crystal thickness. Thus the THz-induced phase retardation accumulated by the probe pulse during its propagation through the crystal is

$$
\Delta \phi\left(t^{\prime}\right)=\phi_{y}\left(t^{\prime}, L\right)-\phi_{z}\left(t^{\prime}, L\right)=f(\theta) \int_{0}^{L} I_{\mathrm{pu}}\left(t^{\prime}+\frac{x}{v_{g}}, x\right) \mathrm{d} x,
$$

with

$$
f(\theta)=\frac{3 \pi b}{4 \lambda_{\mathrm{pr}} n_{\mathrm{pr}}}\left[u-1-(u-3) \sin ^{2} 2 \theta\right] .
$$

This phase retardation is expected to be small compared to 1 .

Then the actual phase retardation measured by our setup through the signal $\Delta I / I$ given by the balanced detection is []]

$$
\begin{aligned}
\frac{\Delta I(\tau)}{I} & =\Delta \phi(\tau) \\
& =f(\theta) \times \int_{-\infty}^{+\infty} \mathrm{d} t^{\prime} \int_{0}^{L} \mathrm{~d} x F_{\mathrm{pr}}\left(t^{\prime}-\tau\right) I_{\mathrm{pu}}\left(t^{\prime}+\frac{x}{v_{g}}, x\right),
\end{aligned}
$$

where $\tau$ is the THz-probe time delay, and $F_{\mathrm{pr}}(t)$ is the probe pulse normalized intensity profile, assumed to be Gaussian with a full width at half-maximum $\tau_{0}$, which is

$$
F_{\mathrm{pr}}(t)=\frac{1}{\sigma_{0} \sqrt{\pi}} \exp \left[-\left(\frac{t}{\sigma_{0}}\right)^{2}\right],
$$

with $\sigma_{0}=\tau_{0} /(2 \sqrt{\ln 2})$.

\section{EXPERIMENTAL RESULTS AND DISCUSSION}

Figure 2 displays a typical phase retardation recorded for the $300 \mu \mathrm{m}$ thick crystal as a function of $\tau$ and at an azimuthal angle $\theta=40^{\circ}$. As expected, we observed that, for both crystal thicknesses, $\Delta \phi$ was small compared with 1 , so that Eq. (ㄷ) 


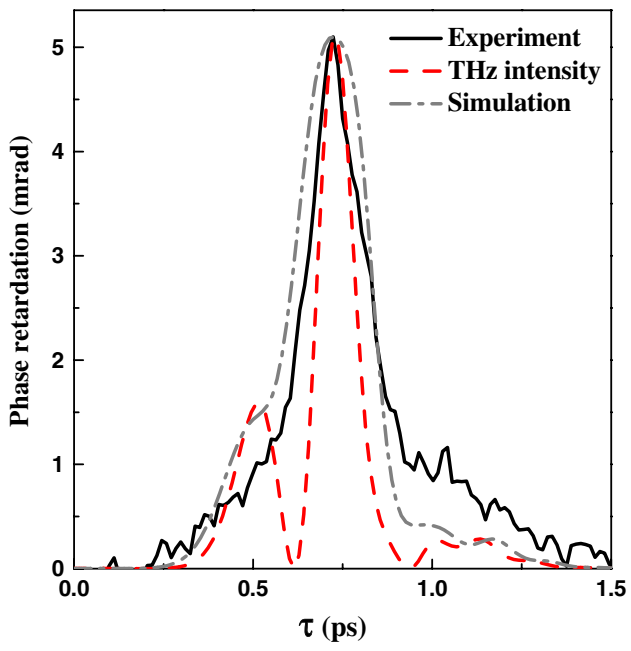

Fig. 2. THz-induced phase retardation recorded in a $300 \mu \mathrm{m}$ thick $\langle 100\rangle \mathrm{GaP}$ crystal for $\theta=40^{\circ}$ (black curve). The dashed red curve corresponds to the input THz "intensity," $I_{\mathrm{pu}}$, normalized to fit the experimental data. The dashed-dotted gray curve corresponds to the phase retardation calculated according to Eq. (5) .

is valid and, accordingly, the peak value of the phase retardation, $\Delta \phi_{\max }$, is expected to behave like

$$
\Delta \phi_{\mathrm{max}} \simeq f(\theta) \times L_{\mathrm{eff}} \times\left(2 E_{\mathrm{THz}}\right)^{2} .
$$

Here $L_{\text {eff }}$ is an effective interaction length introduced to account for the velocity mismatch between the $\mathrm{THz}$ wave and the near-infrared probe pulse in GaP [10]. First let us consider a spectral component of the $\mathrm{THz}$ wave at frequency $\Omega$. Its effective interaction length in GaP is then defined as

$$
L_{\mathrm{eff}}(\Omega)=\left|\int_{0}^{L} \mathrm{~d} x \exp \left[i\left(\frac{\Omega \tilde{n}(\Omega) x}{c}-\frac{\Omega x}{v_{g}}\right)\right]\right|,
$$

where $\tilde{n}$ is the complex refractive index of the crystal in the THz range. Figure 3 shows the ratio $L_{\text {eff }}(\Omega) / L$ computed for

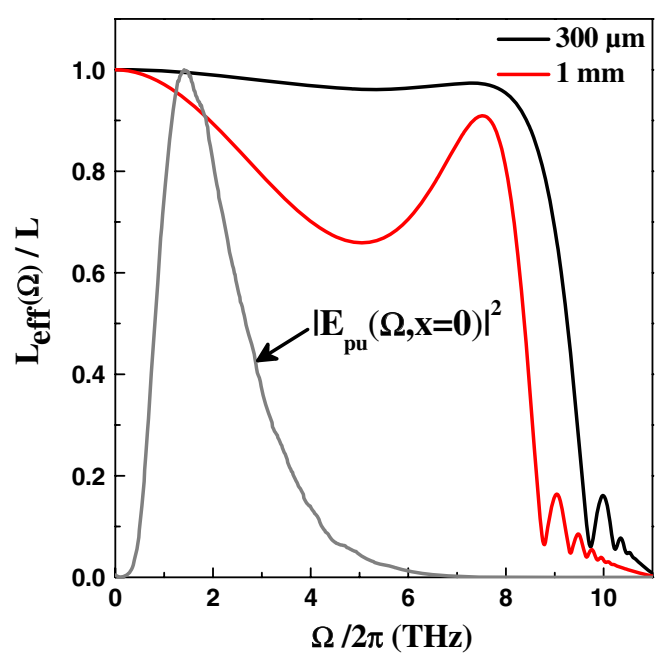

Fig. 3. Evolution of the effective interaction length $L_{\text {eff }}$ versus the frequency $\Omega$ for $L=300 \mu \mathrm{m}$ (black curve) and $L=1 \mathrm{~mm}$ (red curve). The gray curve corresponds to the normalized spectral intensity, $\left|E_{\mathrm{pu}}(\Omega, 0)\right|^{2}$, of the incident $\mathrm{THz}$ wave.
$L=300 \mu \mathrm{m}$ and $1 \mathrm{~mm}$ and for the complex refractive index $\tilde{n}(\Omega)$ given in [11]. Now, by introducing the complex spectrum of the incident THz pulse, $E_{\mathrm{pu}}(\Omega, x=0)=\mathcal{F}\left[E_{\mathrm{pu}}(t, x=0)\right]$ $(\mathcal{F}$ is the complex Fourier transform and $\Omega \geq 0$ ), one can define $L_{\text {eff }}$ as the average of $L_{\text {eff }}(\Omega)$ with respect to the spectral intensity, $\left|E_{\mathrm{pu}}(\Omega, 0)\right|^{2}$, which is

$$
L_{\text {eff }}=\frac{\int_{0}^{+\infty} \mathrm{d} \Omega L_{\mathrm{eff}}(\Omega) \times\left|E_{\mathrm{pu}}(\Omega, 0)\right|^{2}}{\int_{0}^{+\infty} \mathrm{d} \Omega\left|E_{\mathrm{pu}}(\Omega, 0)\right|^{2}} .
$$

Using the spectral intensity given by the electro-optic detection of the THz wave in a $200 \mu \mathrm{m}$ thick $\langle 110\rangle \mathrm{GaP}$ crystal (gray curve of Fig. 3), we found $L_{\text {eff }}=295 \mu \mathrm{m}$ (respectively, $880 \mu \mathrm{m}$ ) for $L=300 \mu \mathrm{m}$ (respectively, $1 \mathrm{~mm}$ ), results that do not deviate significantly from the actual thickness of the crystal. This is explained by Fig. 3: for the thin crystal, $L_{\text {eff }}(\Omega) \simeq L$ over the entire spectral range involved in the experiment, so that $L_{\text {eff }} \simeq L$ after averaging. On the contrary, for the thick crystal, $L_{\text {eff }}(\Omega)$ decreases significantly near $\Omega /(2 \pi)=5 \mathrm{THz}$. However, this "hole" primarily overlaps the tail of the spectral intensity, so that its contribution to the decrease of $L_{\text {eff }}$ in comparison to $L$ is rather small.

Figure $\underline{4}$ confirms the quadratic evolution of $\Delta \phi_{\max }$ with $E_{\mathrm{THz}}$ for both crystals. Moreover, a fit of both curves with the function $A \times E_{\mathrm{THz}}^{2}$ also indicates the proportionality of $\Delta \phi_{\max }$ versus $L_{\text {eff }}$. Indeed, we obtained $A(1 \mathrm{~mm}) / A(300 \mu \mathrm{m})=$ 2.90 , which is close to $L_{\text {eff }}(1 \mathrm{~mm}) / L_{\text {eff }}(300 \mu \mathrm{m})=2.98$.

The experimental angular dependence for a $1 \mathrm{~mm}$ thick crystal is displayed in Fig. 5. It matches rather well with Eq. (4). Note that we observed the same behavior for the $300 \mu \mathrm{m}$ thick crystal, but with a worse signal-to-noise ratio. From the fitting of our data with the function $\Delta \phi_{\max }(\theta)$ given by Eq. (5), we extracted the components of the third-order response function for both crystals. The averaged values of these coefficients are $a=\mathcal{R}_{\text {iiii }}^{(3)}=7.4 \times 10^{-20} \mathrm{~m}^{2} \cdot \mathrm{V}^{-2}$ and $b=\mathcal{R}_{i i j j}^{(3)}=4.0 \times 10^{-20} \mathrm{~m}^{2} \cdot \mathrm{V}^{-2}$. Usually, one is interested in the nonlinear refraction coefficient defined as $n_{2}^{I}=\Delta n / I_{\mathrm{THz}}$, where $\Delta n$ is the change of refractive index due to the Kerr effect and $I_{\mathrm{THz}}=\varepsilon_{0} c n_{\mathrm{THz}} E_{\mathrm{THz}}^{2}$. From the definition of $\Delta n$,

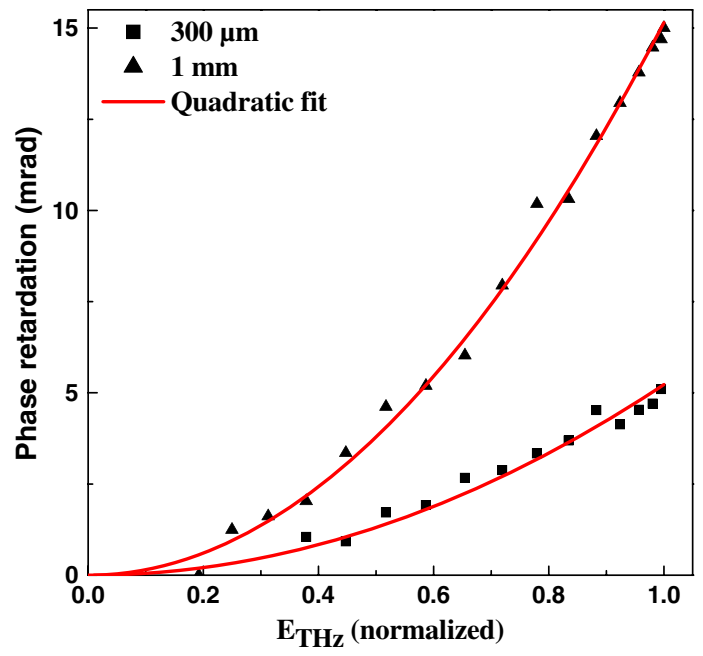

Fig. 4. Peak value of the THz-induced phase retardation with respect to the THz electric field strength $E_{\mathrm{THz}}$ for a $300 \mu \mathrm{m}$ (filled square) and a $1 \mathrm{~mm}$ thick (filled triangle) crystal $\left(\theta=40^{\circ}\right)$. The red curves correspond to a quadratic fit of the experimental data. 


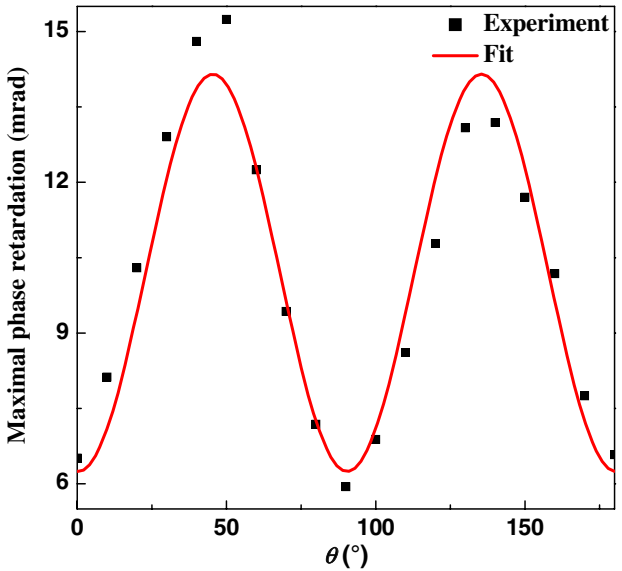

Fig. 5. Azimuthal dependence of the peak value of the THz-induced phase retardation for a $1 \mathrm{~mm}$ thick crystal. The red curve corresponds to a fit of the data by the function $\Delta \phi_{\max }(\theta)=f(\theta) \times L_{\mathrm{eff}} \times\left(2 E_{\mathrm{THz}}\right)^{2}$ $[f(\theta)$ is defined by Eq. ( $\underline{4})]$.

$$
\Delta \phi_{\max }=\frac{2 \pi}{\lambda_{\mathrm{pr}}} \times \Delta n \times L_{\mathrm{eff}}
$$

and Eq. (6), one obtains

$$
n_{2}^{I}(\theta)=\frac{3 b}{2 \varepsilon_{0} c n_{\mathrm{THz}} n_{\mathrm{pr}}}\left[u-1-(u-3) \sin ^{2} 2 \theta\right] .
$$

An average of this expression with respect to $\theta$ then leads to

$$
\left\langle n_{2}^{I}\right\rangle_{\theta}=\frac{3(a+b)}{4 \varepsilon_{0} c n_{\mathrm{THz}} n_{\mathrm{pr}}} .
$$

Assuming that $n_{\mathrm{THz}}=n(1.5 \mathrm{THz})$, we found $\left\langle n_{2}^{I}\right\rangle_{\theta}=$ $1.2 \times 10^{-13} \mathrm{~cm}^{2} \cdot \mathrm{W}^{-1}$. This large value is of the same order of magnitude as the nonlinear refractive coefficient reported in the near-infrared, $n_{2 \mathrm{NIR}}^{I}=6.5 \times 10^{-14} \mathrm{~cm}^{2} \cdot \mathrm{W}^{-1}$ [12].

Finally, we investigated numerically the consistency of the temporal shape of the phase retardation with what is expected from Eq. (5). In our simulation, we took for the incident $\mathrm{THz}$ field the electro-optic signal given by a $200 \mu \mathrm{m}$ thick $\langle 110\rangle \mathrm{GaP}$ crystal and assumed the probe pulse to have an initial duration, $\tau_{0}=60$ fs. We also took into account the dispersion and the absorption for the $\mathrm{THz}$ wave, so that

$$
E_{\mathrm{pu}}\left(t+\frac{x}{v_{g}}, x\right)=2 \times \operatorname{Re}\left\{\mathcal{F}^{-1}\left[E_{\mathrm{pu}}(\Omega, 0) \times T(\Omega, x)\right]\right\},
$$

with $T(\Omega, x)=\exp \left[i\left(\Omega \tilde{n}(\Omega) x / c-\Omega x / v_{g}\right)\right]$. The probe pulse is affected by dispersion as well. Indeed, at a distance $x$ from the entrance of the crystal, the probe pulse duration is broadened by a factor $\beta(x)=\sqrt{1+\left(x / L_{d}\right)^{2}}$, where $L_{d}=\tau_{0}^{2} /\left(k_{\mathrm{pr}}^{\prime \prime} 4 \ln 2\right)$, with $k_{\mathrm{pr}}^{\prime \prime}=\left(\mathrm{d}^{2} k / \mathrm{d} \omega^{2}\right)_{\omega_{\mathrm{pr}}}$ and $k(\omega)=\omega n(\omega) / c$ [1]. Simultaneously, its intensity is divided by the same factor, $\beta(x)$. To account for this phenomenon, we have replaced in Eq. (5) the function $F_{\mathrm{pr}}(t)$ by the following one:

$$
F_{\mathrm{pr}}(t, x)=\frac{1}{\sigma_{p} \sqrt{\pi}} \exp \left[-\left(\frac{t}{\sigma_{p}}\right)^{2}\right],
$$

with $\sigma_{p}=\sigma_{0} \times \beta(x), k_{\mathrm{pr}}^{\prime \prime}$ being calculated thanks to the data of [13]. The simulated temporal shape of $\Delta \phi(\tau)$ is shown in Fig. 2

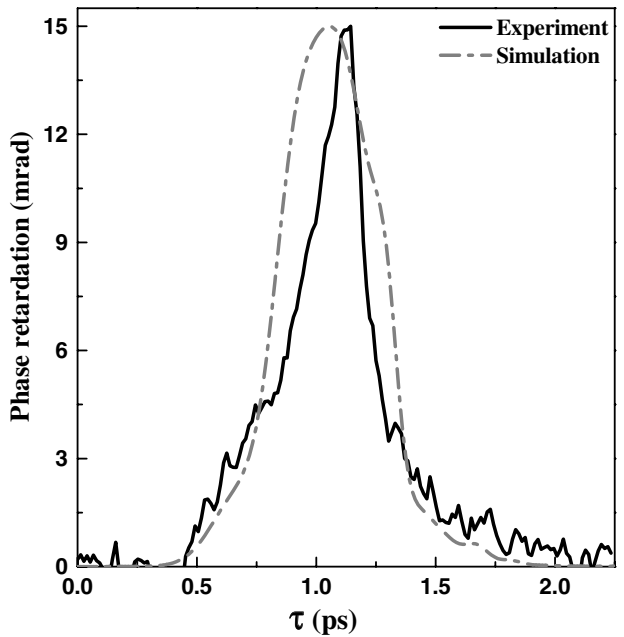

Fig. 6. THz-induced phase retardation recorded in a $1 \mathrm{~mm}$ thick $\langle 100\rangle \mathrm{GaP}$ crystal for $\theta=40^{\circ}$ (black curve). The dashed-dotted gray curve corresponds to the phase retardation calculated according to Eq. (5).

and agrees well with the experiment. For the thin crystal considered here, $\Delta \phi(\tau)$ does not deviate significantly from the input THz pulse "intensity" $I_{\mathrm{pu}}(t, 0)$, it is just slightly broadened by the convolution with $F_{\mathrm{pr}}$. However, for the thick crystal (Fig. 6), we found a temporal width of $\Delta \phi(\tau)$ which is twice as wide as the experimental one, the support of the signal being the same both in the experiment and in the simulation. First of all, we attribute this discrepancy to the fact that we neglected, in our model, the dispersion of the third-order susceptibility of the crystal. Furthermore, unlike the spectrum displayed in Fig. 3, which comes from electro-optic detection in $\mathrm{GaP}$, the spectrum of $\mathrm{THz}$ pulses generated by plasma sources goes as far as $100 \mathrm{THz}$ [14]. Such a spectrum can easily excite the phonon resonance of the GaP residing at $11 \mathrm{THz}$, which, in turn, should probably affect the temporal shape of the Kerr signal. Yet it is noteworthy that these crude approximations do not seem to be detrimental in the modeling of the nonlinear response of a thin crystal, as shown in Fig. 2 .

\section{CONCLUSION}

We have observed a THz-induced Kerr birefringence in gallium phosphide crystals. Thanks to the measurement of the angular dependence of the Kerr signal and the theoretical analysis of the experiment, we have determined the nonzero tensor elements of the third-order response function of this crystal in the THz range. Thus it exhibits a large nonlinear refractive index in this spectral domain. We have also compared the temporal shape of the phase retardation with our model. For a thin crystal, we found overall good agreement between experiment and theory.

\section{ACKNOWLEDGMENTS}

This work has been developed on the COLA platform of the LOMA, supported by Région Aquitaine, FEDER, CNRS, and the University of Bordeaux. The authors acknowledge financial support of the Conseil Régional d'Aquitaine through the project Hi_ter (2011 1603 004). M. C. is grateful for thesis funding from the Direction Générale de l'Armement (DGA) and the Région Aquitaine. 


\section{REFERENCES}

1. J.-C. Diels and W. Rudolph, Ultrashort Laser Pulse Phenomena (Academic, 2006).

2. R. Righini, "Ultrafast optical Kerr effect in liquids and solids," Science 262, 1386-1390 (1993).

3. M. C. Hoffmann, N. C. Brandt, H. Y. Hwang, K. Yeh, and K. A. Nelson, "Terahertz Kerr effect," Appl. Phys. Lett. 95, 231105 (2009).

4. M. Zalkovskij, A. C. Strikwerda, K. Iwaszczuk, A. Popescu, D. Savastru, R. Malureanu, A. V. Lavrinenko, and P. U. Jepsen, "Terahertz-induced Kerr effect in amorphous chalcogenide glasses," Appl. Phys. Lett. 103, 221102 (2013).

5. J. Hebling, K. Yeh, M. C. Hoffmann, and K. A. Nelson, "Highpower THz generation, THz nonlinear optics, and THz nonlinear spectroscopy," IEEE J. Sel. Top. Quantum Electron. 14, 345-353 (2008).

6. D. Turchinovich, J. M. Hvam, and M. C. Hoffmann, "Self-phase modulation of a single-cycle terahertz pulse by nonlinear freecarrier response in a semiconductor," Phys. Rev. B 85, 201304 (2012).

7. M. D. Thomson, M. Kreß, T. Löffler, and H. G. Roskos, "Broadband $\mathrm{THz}$ emission from gas plasmas induced by femtosecond optical pulses: from fundamentals to applications," Laser Photon. Rev. 1, 349-368 (2007).

8. P. N. Butcher and D. Cotter, The Elements of Nonlinear Optics (Cambridge University, 1991).

9. G. Gallot and D. Grischkowsky, "Electro-optic detection of terahertz radiation,” J. Opt. Soc. Am. B 16, 1204-1212 (1999).

10. Q. Wu and X.-C. Zhang, "7 terahertz broadband GaP electrooptic sensor," Appl. Phys. Lett. 70, 1784-1786 (1997).

11. D. Dietze, K. Unterrainer, and J. Darmo, "Dynamically phase-matched terahertz generation," Opt. Lett. 37, 1047-1049 (2012)

12. F. Liu, Y. Li, Q. Xing, L. Chai, M. Hu, C. Wang, Y. Deng, Q. Sun, and C. Wang, "Three-photon absorption and Kerr nonlinearity in undoped bulk GaP excited by a femtosecond laser at $1040 \mathrm{~nm}$," J. Opt. 12, 095201 (2010).

13. F. L. Madarasz, J. O. Dimmock, N. Dietz, and K. J. Bachmann, "Sellmeier parameters for $\mathrm{ZnGaP}_{2}$ and GaP," J. Appl. Phys. 87, 1564-1565 (2000).

14. M. D. Thomson, V. Blank, and H. G. Roskos, "Terahertz white-light pulses from an air plasma photo-induced by incommensurate two-color optical fields," Opt. Express 18, 2317323182 (2010). 